\title{
Satellite Gamelan: microtonal sonification using a large consort of mobile phones
}

\author{
Greg Schiemer \\ composer, instrument-builder and software developer \\ greg.schiemer@protonmail.com
}

\begin{abstract}
This paper describes an approach to sonification based on an iPhone app created for multiple users to explore a microtonal scale generated from harmonics using the combination product set method devised by tuning theorist Erv Wilson. The app is intended for performance by a large consort of hand-held mobile phones where phones are played collaboratively in a shared listening space. Audio consisting of handbells and sine tones is synthesised independently on each phone. Sound projection from each phone relies entirely on venue acoustics unaided by mains-powered amplification. It was designed to perform a microtonal composition called Transposed Dekany which takes the form of a chamber concerto in which a consort of players explore the properties of an microtonal scale. The consort subdivides into families of instruments that play in different pitch registers assisted by processes that are enabled and disabled at various stages throughout the performance. The paper outlines Wilson's method, describes its current implementation and considers hypothetical sonification scenarios for implementation using different data with potential applications in the physical world.
\end{abstract}

\section{Author Keywords}

collaborative sonification, mobile performance, microtonal composition, software instrument, pocket gamelan

\section{INTRODUCTION}

Ever since the first ICAD more than two decades ago the art of auditory display has become a tool to interpret complex data in many forms. [1] Its applications range from the use of audio signals to represent various kinds of natural phenomena such as tidal data collected over many years using conventional record keeping methods [2] to the design of air traffic control consoles for 'receiving, analyzing and acting upon complex information in a timely manner'. [3] The Listening to the Mind Listening project also showed how interpretations of the same data set by different sonification designers can vary in their musical characteristics [4] In its current form it is possible to argue that Transposed Dekany, the work described in this paper, is composition more than sonification. Stephen Barrass clarifies the distinction between the two:

When the primary intention of the composer shifts to the revelation of the source, the work crosses into the realm of sonification. With this crossing over comes a question of whether the listener can also understand the composer's intention to produce more than an experience of the music itself. [5]

Hermann describes Model-Based Sonification as a transformation from data to sound where

data is used to build an instrument or sound-capable object, while the playing is left to the user.

This almost describes the Satellite Gamelan, the app I created to perform Transposed Dekany, and potentially satisfies all four conditions in Hermann's definition of sonification - namely, that the sound reflects objective properties, that the transformation of data is systematic, that sonification is reproducible and that it can be used with different data.

\section{PUBLIC SOUND}

The most common presentation for auditory display involves sound projected from a fixed location such as stereo or multi-channel speakers or binaural headphones. With the improved audio signal processing capabilities and widespread uptake of mobile phone technology there is now an alternative framework for public listening which I call collaborative sonification.

Collaborative sonification allows many listeners to present and interact with complex data in a shared acoustic environment. Sound is projected via a large malleable speaker array consisting of handheld battery-powered mobile phone speakers. Each phone becomes an independent sound source like any conventional hand-held musical instrument which results in the creation of ensemble sound albeit from a consort of electronic sound sources. The ideal built environment for such a presentation is a performance venue with high ceilings and reflective acoustics. Sound projection is no longer entrusted to one listener positioned at an electronic mixing desk trying (or perhaps not even bothering) to second-guess what listeners might expect to hear.

There are obvious limitations in projecting sound from a miniature loudspeaker with the power limitations and frequency response of a speaker phone. As with conventional consorts of viols, recorders or voices, strength comes from the size of the ensemble. While it is possible to produce audio covering the audible spectrum and project audio using mains powered speaker tether the phone to project I have chosen to work within the frequency response limitations of the speaker phone.

Vickers noted that using 'organising principles of tonal music' to present data can 'result in more aesthetically-coherent sonifications'. [6] If those principles were extended to include music with a nonequally tempered pitch spectrum based on harmonic ratios could this not also provide 'a system that is more open to reading than it is a musical style that is recognized as such'?

My focus has not been what to do about a limited frequency response, but rather, what to do with frequencies produced within a limited range of four octaves. In fact the focus on frequency is even more specific: it is about unequal relationships that occur between pitches of a scale derived from pure harmonics. In the ideal acoustic environment a mobile phone speaker will clearly reproduce signals derived from pure harmonics played at the appropriate frequency. For that reason the app does not use sampled audio but synthesises bell tones and chorus tones by adding sines tones. These are tuned in a variety of ways using a simple algorithm based on harmonics.

The world's musical traditions have many such scales where the size of intervals, i.e. the gap between two notes, is defined by points on the harmonic series. One of the most common scales are pentatonic. Some scales will sound more recognisably pentatonic than others that are tuned using different harmonics; this is especially true for audiences conditioned to hearing a pentatonic scale played on the black notes of a conventional music keyboard.

The Satellite Gamelan app was never intended to sonify anything more than abstract data i.e. harmonic numbers. Nevertheless it offers a rich and elegantly variable palette of harmonic flavours derived from numbers available in the harmonic series. 


\section{MOBILE COLLABORATION}

The work has precedents in other battery powered mobile events such as Iain Mott's Sound Mapping in 1998 [7] and Golan Levin's Dialtones (A Telesymphony)[8] in 2001. The latter involved active participation by a concert audience. Mobile phones became a multichannel sound system through which triggered sequences of ringtones are played. Mobile phone ensembles have sprung up at Stanford, Michigan and Helsinki Universities using apps that synthesise audio with players wearing battery-powered amplifierspeakers [9]. These, along with the Satellite Gamelan, are pre-dated by earlier mobile projects I developed for j2me phones [10] and purpose-built battery powered analogue circuitry called UFOs [11].

\section{MICROTONAL TUNING}

The app also builds on the microtonal legacy of composer, instrument-builder and theorist Harry Partch whose work recognised and celebrated the diversity of tuning that lies outside the western concert tradition [12]. Each phone is tuned to a dekany, a 10-note scale generated from harmonics using a method devised by tuning theorist and instrument-builder Erv Wilson.

Wilson theories include a system of keyboard mapping that provides a broader historical perspective for Partch's work and a trajectory that embraces both experimental and traditional tuning [13]. One of Wilson's tuning theories, known as combination product sets (CPS) is a way to generate many scales of various size and harmonic flavour.

\subsection{Dekany}

The dekany is a CPS scale where pitches are generated by multiplying combinations of two harmonics from a set of five harmonics: $1,3,7,9$ and 11 .

Table 1 shows the relationship between pitches of the dekany and its five harmonic generators. Pitches are described in cents and ratios. Note that unison - 0 cents or $1 / 1-$ is missing from the scale, the salient feature of CPS scales; nevertheless, for convenience, rational scale pitches are defined with reference to unison. In a CPS scale the numerator represents a product while the denominator represents the missing unison, or some octave above it.

Table1. Pitches of the 1-3-7-9-11 dekany with five generators

\begin{tabular}{|c|c|c|c|c|c|c|c|}
\hline & cents & ratios & 1 & 3 & 7 & 9 & 11 \\
\hline 1 & 53.273 & $33 / 32$ & & 3 & & & 11 \\
\hline 2 & 203.910 & $9 / 8$ & 1 & & & 9 & \\
\hline 3 & 320.144 & $77 / 64$ & & & 7 & & 11 \\
\hline 4 & 470.781 & $21 / 16$ & & 3 & 7 & & \\
\hline 5 & 551.318 & $11 / 8$ & 1 & & & & 11 \\
\hline 6 & 701.955 & $3 / 2$ & 1 & 3 & & & \\
\hline 7 & 755.228 & $99 / 64$ & & & & 9 & 11 \\
\hline 8 & 905.865 & $27 / 16$ & & 3 & & 9 & \\
\hline 9 & 968.826 & $7 / 4$ & 1 & & 7 & & \\
\hline 10 & 1172.736 & $63 / 32$ & & & 7 & 9 & \\
\hline
\end{tabular}

The 1-3-7-9-11 dekany has two interleaved scales that are recognisably pentatonic. Their pitches are separated by two intervals, one small and one large, just like pentatonic scales played on an equal tempered keyboard. Pitches of the odd numbered pentatonic scale are separated by intervals of 231.174 and 266.871 cents while pitches of the even numbered pentatonic scale are separated by intervals of 213.598 and 284.447 cents; by comparison, pitches of pentatonic scales played on a conventional equal tempered 12-tone keyboard are separated by intervals of 200 and 300 cents.
Each pentatonic scale represents a different harmonic flavour, a result of differences in the size of their small and large intervals. These flavours produce strong consonance when heard separately and strong dissonance when heard simultaneously, a dissonance and consonance stronger than any produced in 12-tET. By organising the 1-3-7-9-11 dekany as two pentatonic scales, I expected to make it easier for musicians to focus on pitches of one scale and selectively ignoring interference from pitches of the other.

This assumption can be tested in a laboratory. Using randomly selected tones interleaved with tones of a familiar melody, Alan Bregman demonstrates how auditory streaming makes it possible for listeners to segregate a stream of melodic tones from interfering tones that camouflage the melodic stream [14].

The 1-3-7-9-11 dekany has two scales each with a different melodic contour. Each tends to camouflage the other and each are recognisably pentatonic, a property related to the harmonic generators used to create the dekany.

\subsection{Transposition}

Transposition is a process for playing a scale in a different register starting on each note of the original scale. On a 12-tone equaltempered keyboard, a transposed scale always fall on other notes on the keyboard. By contrast, transposing a scale with pitches that are generated from harmonics will result in additional harmonically related pitches that are not present in the original scale.

New harmonic flavours are created when different transpositions of the dekany are heard simultaneously. In performance players are divided into five groups, each playing in a different transposition.

The five transpositions are shown in Table 2, rearranged as interleaved odd and even pentatonic scales represented here in cents. Transposition results in 26 new pitches. Each transposition has some pitches in common with other transpositions creating harmonic connections between them.

\begin{tabular}{|c|c|c|c|c|c|c|}
\hline & dekany & t1 & t2 & t3 & t4 & t5 \\
\hline \multicolumn{2}{|c|}{ odd pentatonic } & सE8.278 & +320.144 & +551.318 & +755.228 & +968.826 \\
\hline 1 & 53.273 & 106.546 & 373.417 & 604.591 & 808.501 & 1022.099 \\
\hline 3 & 320.144 & 373.417 & 640.288 & 871.462 & 1075.372 & 88.970 \\
\hline 5 & 551.318 & 604.591 & 871.462 & 1102.636 & 106.546 & 320.144 \\
\hline 7 & 755.228 & 808.501 & 1075.372 & 106.546 & 310.456 & 524.054 \\
\hline 9 & 968.826 & 1022.099 & 88.970 & 320.144 & 524.054 & 737.652 \\
\hline \multicolumn{2}{|c|}{ even pentatonic } & +208.910 & +470.781 & +701.955 & +905.865 & +1172.736 \\
\hline 2 & 203.910 & 407.820 & 674.691 & 905.865 & 1109.775 & 176.646 \\
\hline 4 & 470.781 & 674.691 & 941.562 & 1172.736 & 176.646 & 443.517 \\
\hline 6 & 701.955 & 905.865 & 1172.736 & 203.910 & 407.820 & 674.691 \\
\hline 8 & 905.865 & 1109.775 & 176.646 & 407.820 & 611.73 & 878.601 \\
\hline 10 & 1172.736 & 176.646 & 443.517 & 674.691 & 878.601 & 1145.472 \\
\hline
\end{tabular}

Table2. 1-3-7-9-11 dekany with five transpositions (in cents)

\section{MOBILE MUSIC}

\subsection{Satellite Gamelan}

The Satellite Gamelan is an app for eighty players. It configures a hand-held phone as an instrument that is played as part of a performing ensemble. The instruments are easy to play, quick to learn and enable a large consort of players to collaborate in the discovery of a new microtonal language. In the process of using the app players perform a composition called Transposed Dekany,. 


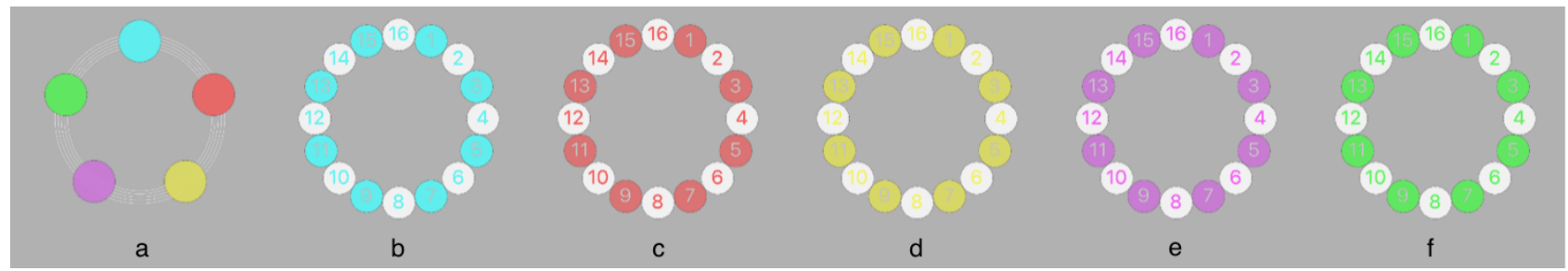

Figure 1. The left view [a] appears when the app is launched. Players select a family by touching 1-of-5 five coloured buttons. This takes them to another view [b, c, d, e or f] where they select 1-of-16 player settings. Each player has a different setting.

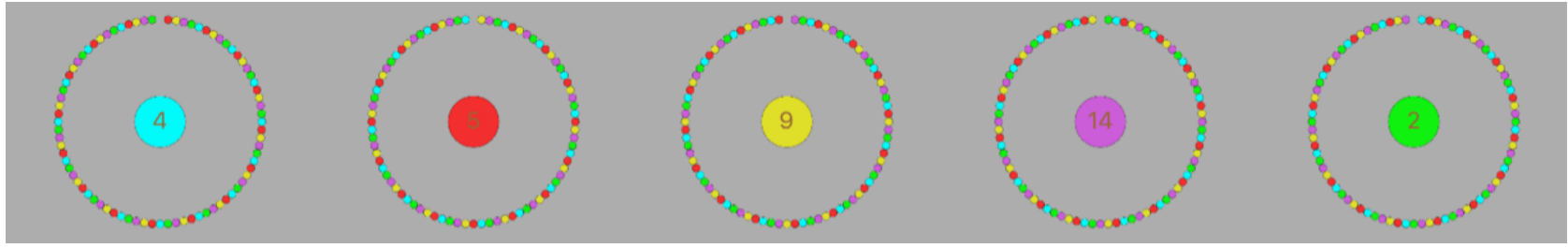

Figure 2. These views appear once players have selected player settings. Players wit for the rest of the consort to make their selection. On an agreed hand signal sign from a designated lead player every player hits the centre button on their screen.

\subsection{Instrument Configuration and Tuning}

Each player configures their phone as a mobile instrument that can be played in one of two ways. Firstly players can shake it like a handbell to produce bell sounds. Alternatively, players can touch buttons to produce a chorus of sine waves.

The full consort subdivides into five families each consisting of sixteen players. Players must first decide what part they will play in the consort, i.e. what family they join and which player they will be. Players then configure their instruments by selecting the family; this is done by touching one of the coloured buttons shown in Figure 1a; then selecting a player number; by touching one of sixteen buttons that appear as rosettes in Figures 1b, 1c, 1d, 1e or 1f.

Each family is tuned to a different scale transposition. This is selected automatically when players join a family (see Figure 1a). Once selected, an instrument remains tuned that way until the end of the performance. Selecting the player number chooses one of sixteen pitch and octave settings available within each family. Settings are uniquely assigned to each player. For example, every player has two bells each with its own pitch; every player has a pallet of chorus sounds played in uniquely assigned pitch and octave combinations.

\subsection{Composition - Transposed Dekany}

Once instrument configuration is complete, the app presents a screen view with a button surrounded by small circles as shown in Figure 2. All players are required to hit the centre button together. This starts a programmed sequence of states that automatically enables or disables the user interface during various states throughout the performance of Transposed Dekanies. The composition has 31 states in total as shown in Figure 3; the duration of each state is 24 "; the total duration is 12 ' 24 '.

The sequence of states generates every combination of the five families. Every family has one state where those players are heard on their own. There is also one state in which every family is heard playing together. Within those boundaries every combination of two, three or four families playing together is heard once and once only.
The sequence is displayed as an animated graphic score that updates whenever a state changes allowing players to monitor their progress in relation to their own family members and other families.

In any given state even numbered players enabled in every family create bell sounds while odd numbered players create chorus sounds. On alternate states players switch roles, i.e. odd players playing bells instead of chorus and vice versa.

Bell tones are played by shaking the phone like a conventional handbell; the centre circle is the bell clapper. Chorus tones are played by touching one of five points on the perimeter circle, or bell rim. These tones are synthesised using some of the first software instruments created by Jean-Claude Risset at Bell Labs [16].

Cues to play bell are shown in Figure 4a (odd) and $4 \mathrm{~b}$ (even); the number in the centre is the selected player number. Cues to play chorus sounds are shown in Figures 4c for playing odd numbered notes of the dekany and $4 \mathrm{~d}$ for playing even numbered notes. The animated graphic score is shown below the cue in each figure.
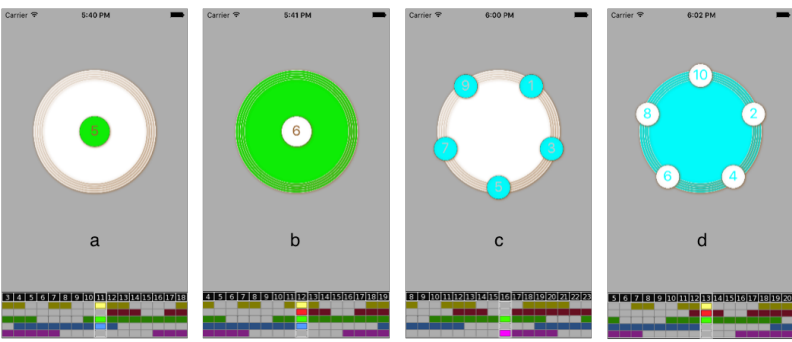

Figure 4. Odd and even bell cues (a,b) and chorus cues (c,d)

When the cue appears on the screen players may respond in their own time by ringing bells or playing chorus sounds within each 24 second window. Bell and chorus decay times are calculated on the fly from the start of the triggered event. They are timed to last until the end of the final active state for that family of instruments or until another event is initiated by the player, whichever occurs first.

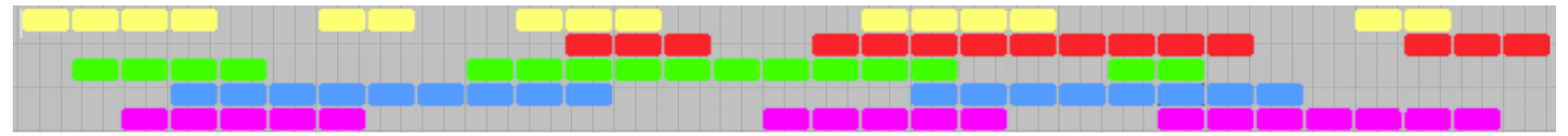

Figure 3. The 5-bit sequence of 31 states enables every phone in each different family. Duration of each state is 24 seconds. 


\section{EXTENDED SCENARIOS}

In its current form the app is a collaborative tool for manipulating a specific abstract data set, namely, the 5 harmonics that generate the scale. With changes the app could include the use of physical data.

\subsection{Harmonic possibilities}

Wilson's CPS method can generate many different dekanies scale each with its own specific harmonic flavour. To do this one might change the harmonics 1379 and 11 to another set of values e.g. 13 713 and 17. Increasing the number of harmonic generators would also produce scales larger than 10 notes per octave.

With further modification the app would allow a user to change harmonics using a slider or touch menu to select new values; with a looped note sequence playing, this would allow a player to make a seamless transition from one harmonic flavour to the next. With further modification to the app, changes made by a user on one phone would also be broadcast to other phones.

Significantly, any change in the harmonic flavour is instantly recognisable irrespective of the musical expectations of the listener.

\subsection{Bicycle flotilla}

The app would lend itself to an event involving a flotilla of cyclists each using an iPhone as the mobile sound source, i.e. without headphones. The event would be an extension of the first Concert on Bicycles in 1983 when about 130 cyclists using ghetto-blasters tuned to a 1-hour broadcast radio program and cycled round Lake Burley Griffin, Canberra; half the cyclist travelled in a clockwise direction, the other half in a counter-clockwise direction, thereby passing one another at double the normal bicycle speed. The mono broadcast was transformed by the mass movement of multiple sound sources to create spatial artefacts discernible only to participating listeners. [16]

In this scenario participants install the app prior to the event. Instead of a ghetto-blaster, cyclists fasten an iPhone to their bicycle frame thereby making it responsive to accelerometer data. With some modification to the app, the handbell becomes a bicycle bell that responds to corrugations in the surface of the track while GPS data transforms the harmonic properties as cyclists enter new terrain.

\section{CONCLUSIONS}

The Satellite Gamelan app was first used on Nov 302012 as part of the Space Time Concerto Competition. On that occasion the performance involved a hook up of several concert venues spanning several continents and interconnected via a satellite link. The app has since been modified to support 80 players in the same venue. [17]

My ultimate objective is to take advantage of venue acoustics enjoyed by performers of conventional concert music. The Satellite Gamelan app used by a large ensemble can augment a standard contemporary concert program. For an established professional orchestra or large choir the economics are straight forward. Transposed Dekany is easy to play and quick to learn. It is currently available as an app that runs on a phone widely used by many musicians. For an existing ensemble of seasoned players the work can be made concert-ready in a single one-hour rehearsal. A performance lasts less than thirteen minutes, requires no special concert amplification and every instrument can be set up by its player without technical support. The missing ingredient so far is a conductor with the vision to convince eighty musicians to put their regular instrument aside (or rest their vocal chords) in order to present a new kind of chamber concerto where 'playing is left to the user' [5] yet executed to the highest standards of ensemble musicianship.

Clearly the problem lies not with the definition of sonification as this has evolved in the ICAD community but rather with limitations we place on what constitutes the 'organising principles of tonal music'. [6] That is a problem I have always had , and will probably continue to have, with music in general.

\section{ACKNOWLEDGMENTS}

I wish to acknowledge Etienne Deleflie for developing the templates that brought me up to speed with Objective C; and Mark Havryliv who helped troubleshoot maths code for Risset bell synthesis; and thanks to the reviewers whose comments helped focus this paper.

\section{REFERENCES}

1.Kramer, G, and Walker, B. "Sound science: Marking ten international conferences on auditory display". in Journal of ACM Transactions on Applied Perception (TAP) Volume 2 Issue 4, October 2005 pp. 383-388

2. Marrin, D. 'Infrasonic Sources in the Environment: Oceanic, Atmospheric and Terrestrial' in Proceedings of Acoustics 2004, November 3-5 pp. 1-7

3. Cabrera, D., Ferguson, S. and Laing, G. "Considerations arising from the development of auditory alerts for air traffic control consoles" in Proceedings of the 12th International Conference on Auditory Display, London, UK, June 20-23, 2006 pp. 242-245

4. Barrass, S. Whitelaw, M. and Bailes, F. "Listening to the Mind Listening: An Analysis of Sonification Reviews, Designs and Correspondences" in Leonardo Music Journal Vol. 16, Noises Off: Sound Beyond Music (2006), pp. 13-19

5. Hermann, T. "Taxonomy and Definitions for Sonification and Auditory Display", in Proc. 14th Int. Conference on Auditory Display (ICAD), Paris, France, June 24-27, 2008.

6. Vickers, P. and Hogg, B. "Sonification ab- straite/sonification concrete: An 'æsthetic perspective space' for classifying auditory displays in the ars musica domain," in ICAD 2006 - The 12th Meeting of the International Conference on Auditory Display, London, UK, June 20-23 2006, pp. 210-216.

7. Mott, I. (with collaborators Raszewski and Sosnin) http:// www.sounddesign.unimelb.edu.au/web/biogs/P000329b.htm

8. Levin, G. et al. "Dialtones (A Telesymphony)" 2001 http:// www.flong.com/projects/telesymphony/index.html/retrieved January 32016

9. Wang, G., Essl, G. and Penttinen, H. "Do mobile phones dream of electric orchestras ?" Proceedings of the International Computer Music Conference (ICMC-08). August 2008

10. Schiemer, G. and Havryliv, M. "Pocket Gamelan: Tuneable trajectories for flying sources in Mandala 3 and Mandala 4". Proceedings of the 2006 International Conference on New Interfaces for Musical Expression (NIME06) June 2006 pp. 37-42

11.Jenkins, J. 22 Australian Composers 1988 (21. Greg Schiemer) http:/www.rainerlinz.net/NMA/22CAC/TOC.html retrieved April 302016

12.Partch, H. "Genesis Of A Music: An Account Of A Creative Work, Its Roots, And Its Fulfillments" Da Capo Press, 1979

13. Narushima, T. "Mapping the microtonal spectrum using Erv Wilson's Generalised Keyboard" PhD Thesis (2012) Routledge Oxford June 2016

14. Bregman, A. Auditory Stream Analysis No.5. Segregation of a melody from interfering tones http://webpages.mcgill.ca/staff/ Group2/abregm1/web/downloadstoc.htm\#05 retrieved April 28 2016

15 .Risset, J. Introductory Catalogue of Computer Synthesised Sounds, Bell Telephone Labs Murray Hill 1969

16. Schiemer, G.. (1994). Interactive Radio. Leonardo Music Journal, 4, 17-22

17. Schiemer, G. Satellite Gamelan (2012) concept video created for submission in the Space Time Concerto competition https:// www.youtube.com/watch? $\mathrm{v}=$ gfaZly6dhQA retrieved January 2 2016 